\title{
Diabetic Retinopathy study via metabolism improvements and chronic diseases medical conditions control of HbA1C, SBP, and Triglycerides (GH- Method: math-physical medicine)
}

Gerald C. Hsu

EclaireMD Foundation, USA

\section{Introduction}

The author used his own medical data to investigate the impact on his diabetic retinopathy condition and its risk probability percentage or hazard ratio (HR) over a period of 7-years.

\section{Methods}

The author suffered from type 2 diabetes (T2D) since 1996. By year 2010, he experienced cardiovascular disease, renal complications, bladder infection, foot ulcer, thyroid disorder, and vision problems. In July of 2010, three of his physicians warned him that he would have three to five remaining years to live. Therefore, he decided to study and research diabetes and its complications in order to save his own life. For the past 10 years, he has spent 30,000 hours on endocrinology with a specialty in diabetes and food nutrition.

\section{The following timetable illustrates the focused area of each} stage:

- 2000-2013 - Self-study diabetes and food nutrition, developing a data collection and analysis software

- 2014 - Develop a mathematical model of metabolism, using engineering modeling and advanced mathematics

- 2015 - Weight \& FPG prediction models, using neuroscience

- 2016 - PPG \& HbA1C prediction models, using optical physics, artificial intelligence (AI), and neuroscience

- 2017 - CVD and Stroke research, using segmentation analysis and pattern analysis

- 2018 - Complications due to micro-vascular research, for example, renal, bladder, and foot

- 2019 - CGM glucose big data analysis, using wave theory, energy theory, frequency domain analysis, quantum mechanics, and AI

- 2020 - Geriatrics, Longevity, Diabetic Retinopathy, Diabetic Hyperthyroidism, linkage between Metabolism and Immunity.

\author{
Correspondence author \\ Gerald C Hsu, \\ EclaireMD Foundation \\ USA
}

Submitted : 06 June 2020 ; Published : 26 June 2020
To date, he has collected $\sim 2$ million data regarding his medical conditions and lifestyle details. He has written 246 medical papers and made $\sim 120$ presentations at $\sim 70$ international medical conferences.

Under the leadership of American Diabetes Association (ADA), a group of 14 authors quoted 137 references to publish a long editorial article, "Perspectives in Diabetes: Diabetic Retinopathy, Seeing Beyond Glucose-Induced Microvascular Disease" [1].

\section{Here is an excerpt:}

The diabetic retinopathy remains the leading cause of vision impairment and blindness. The risk of developing vision loss from diabetes is predicted to double over the next three decades.

The fundamental functions of retina are to capture photons, convert the photochemical energy into electric energy, integrate the resulting action potentials, and transmit them to the occipital lobe of the brain, where they are deciphered and interpreted into recognizable images. Although the retina is easily visible, it is, ironically, the only major tissue affected by diabetes that cannot be biopsied in human.

Established neurobiological principles can inform us how diabetes impairs vision, and metabolism knowledge may lead to new treatments. Retina physiology may underlie its vulnerability to diabetes. The combination of high metabolic demand and minimal vascular supply may limit the inner retina's ability to adapt to the metabolic stress of diabetes. The pathogenesis of diabetic retinopathy includes glucosemediated microvascular damage. Although micro vascular changes are undeniably integral to retinopathy, the retina is actually a vascularized neural tissue, not a network of blood vessels. Diabetic retinopathy involves more than elevated 
glucose and micro vascular lesions. Plenty of evidences for neural retinal involvement in diabetic retinopathy have already been presented.

To the best of our knowledge, there is no evidence that a primary, selective defect in vascular cells is sufficient to cause diabetic retinopathy. Clearly, it is essential to treat both the vascular and neural elements of the retina to preserve vision.

Excess glucose (elevated HbA1C) is the primary culprit in the development and progression of diabetic retinopathy. However, disordered lipid (especially triglycerides) and protein metabolism are also linked to the central biochemical abnormality in all forms of impaired insulin action.

In reference 2, a paper titled "Risk factors associated with progression to referable retinopathy" was written and presented by J. J. Smith, D. M. Wright, P. Scanlon, \& N. Lois:

This study was conducted in a dynamic cohort of 2,770 type 2 diabetes patients, recruited between April 2005 and July 2013 ( $\sim 8$ years) in Ireland. In this diabetic retinopathy paper, the authors demonstrated that Higher current values of HbA1C, systolic blood pressure (SBP), and triglycerides were associated with increased risk of referral diabetic retinopathy.

Based on the findings from the long editorial article, the author conducted a study on his own diabetic retinopathy development and progression for the past 7-years (2013-2019). He has collected and further calculated the following data categories associated with his own medical conditions:

Weight: 2 body weight, BMI

Glucose: 1 FPG, 3 PPG, 1 daily A1C

Blood Pressure: SBP, DBP, pulse

Lipid: Triglycerides, HDL, LDL

The data for the top three categories were collected (weight, glucose, and BP) or calculated (i.e. HbA1C, BMI and predicted glucoses) on a daily basis. However, his lipid results were obtained from 22 hospital lab-tests with an average testing period of every four months.

Initially, he listed his lab-tested Triglycerides data, and then extracted data from both measured $\mathrm{HbA1C}$ and SBP from his stored database to match the actual dates of his lab-test for triglycerides (a total of 22 dates). In summary, 38,385 data of these 2,555 days (7-years) were used in this study.

Next, he selected the following medical conditions as his baseline conditions (i.e. "normal conditions") for his risk probability analysis.

Triglycerides: $150 \mathrm{mg} / \mathrm{dL}$

SBP: 120

HbA1C: $6.0 \%$

Finally, he applied a linear regression analysis model with 7 different cases to conduct his numerical analysis.

In addition, he also applied Cox proportional-hazards regression model to conduct one more set of calculation. The Cox Hazard Model can be expressed as follows:

$h(t)=$

$h 0(t) * \exp (b 1 \times 1+b 2 \times 2+\ldots+b n x n)$

Where $\mathrm{h}(\mathrm{t})$ is the expected hazard at time $\mathrm{t}, \mathrm{h} 0(\mathrm{t})$ is the baseline hazard and represents the hazard when all of the predictors (or independent variables) $x 1, x 2 \ldots x n$ are equal to zero. Notice that the predicted hazard (i.e., $h(t))$, or the rate of suffering the event of interest in the next instant, is the product of the baseline hazard $(\mathrm{h} 0(\mathrm{t}))$ and the exponential function of the linear combination of the predictors. Thus, the predictors have a multiplicative or proportional effect on the predicted hazard.

\section{Results}

Figure 1 shows his records of Triglyceride, SBP, and HbA1C, where his SBP curve is expressed with 90-days moving average data.

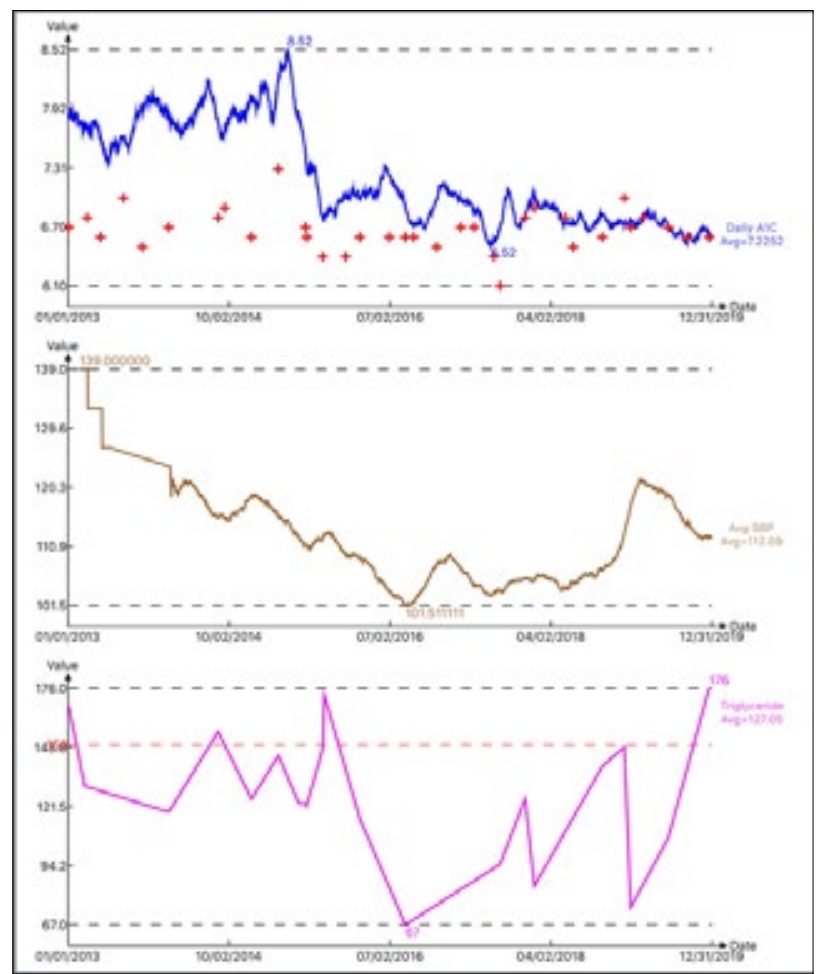

Figure 1: HbA1C, SBP, Triglycerides during 2013 - 2019

After he matches the dates of both $\mathrm{HbA1C}$ and SBP with the actual lab-test dates of Triglycerides, he then normalized those daily medical values by using baseline or normal conditions. These original data and normalized results are shown in Figure 2. 


\begin{tabular}{|c|c|c|c|c|c|c|c|}
\hline Original Data & & & & Standard \% & 150 & 120 & 6.0 \\
\hline Date & Trig. & SBP & HbA1C & Date & Trig. & SBP & HbA1C \\
\hline $1 / 4 / 13$ & 168 & 139 & 7.83 & $1 / 4 / 13$ & 1.12 & 1.16 & 1.31 \\
\hline $3 / 8 / 13$ & 131 & 139 & 7.74 & $3 / 8 / 13$ & 0.87 & 1.16 & 1.29 \\
\hline $2 / 5 / 14$ & 119 & 124 & 7.86 & $2 / 5 / 14$ & 0.79 & 1.03 & 1.31 \\
\hline $8 / 20 / 14$ & 156 & 116 & 7.77 & $8 / 20 / 14$ & 1.04 & 0.97 & 1.30 \\
\hline $12 / 30 / 14$ & 125 & 119 & 8.02 & $12 / 30 / 14$ & 0.83 & 0.99 & 1.34 \\
\hline $4 / 16 / 15$ & 145 & 116 & 8.20 & $4 / 16 / 15$ & 0.97 & 0.97 & 1.37 \\
\hline $7 / 6 / 15$ & 123 & 112 & 7.85 & $7 / 6 / 15$ & 0.82 & 0.93 & 1.31 \\
\hline $8 / 4 / 15$ & 123 & 111 & 7.39 & $8 / 4 / 15$ & 0.82 & 0.93 & 1.23 \\
\hline $10 / 9 / 15$ & 148 & 112 & 6.79 & $10 / 9 / 15$ & 0.99 & 0.93 & 1.13 \\
\hline $10 / 15 / 15$ & 173 & 113 & 6.78 & $10 / 15 / 15$ & 1.15 & 0.94 & 1.13 \\
\hline $3 / 4 / 16$ & 116 & 109 & 6.98 & $3 / 4 / 16$ & 0.77 & 0.91 & 1.16 \\
\hline $9 / 1 / 16$ & 67 & 102 & 6.91 & $9 / 1 / 16$ & 0.45 & 0.85 & 1.15 \\
\hline $9 / 12 / 17$ & 95 & 104 & 6.81 & $9 / 12 / 17$ & 0.63 & 0.87 & 1.14 \\
\hline $12 / 20 / 17$ & 125 & 106 & 6.89 & $12 / 20 / 17$ & 0.83 & 0.88 & 1.15 \\
\hline $1 / 26 / 18$ & 85 & 105 & 7.01 & $1 / 26 / 18$ & 0.57 & 0.88 & 1.17 \\
\hline $10 / 22 / 18$ & 140 & 107 & 6.84 & $10 / 22 / 18$ & 0.93 & 0.89 & 1.14 \\
\hline $1 / 18 / 19$ & 149 & 113 & 6.74 & $1 / 18 / 19$ & 0.99 & 0.94 & 1.12 \\
\hline $2 / 12 / 19$ & 75 & 117 & 6.77 & $2 / 12 / 19$ & 0.50 & 0.98 & 1.13 \\
\hline $7 / 11 / 19$ & 107 & 109 & 6.72 & $7 / 11 / 19$ & 0.71 & 0.91 & 1.12 \\
\hline $12 / 20 / 19$ & 176 & 113 & 6.64 & $12 / 20 / 19$ & 1.17 & 0.94 & 1.11 \\
\hline Avarage & 127 & 114.3 & 7.23 & Avarage & 0.85 & 0.95 & 1.20 \\
\hline
\end{tabular}

Figure 2: Original data and normalized value of $\mathrm{HbA1C}, \mathrm{SBP}$, Triglycerides of selected dates during 2013 - 2019

The author could not find the contribution margins (i.e. weighting factors) related to these three primary factors of diabetic retinopathy study (i.e. A1C, SBP, and Triglycerides) from the external references; therefore, he decided to conduct a sensitivity analysis by using a range of possible weighting factors. Figures 3, 4, 5, and 6 reflect the operational results and his seven different cases of weighting factors.

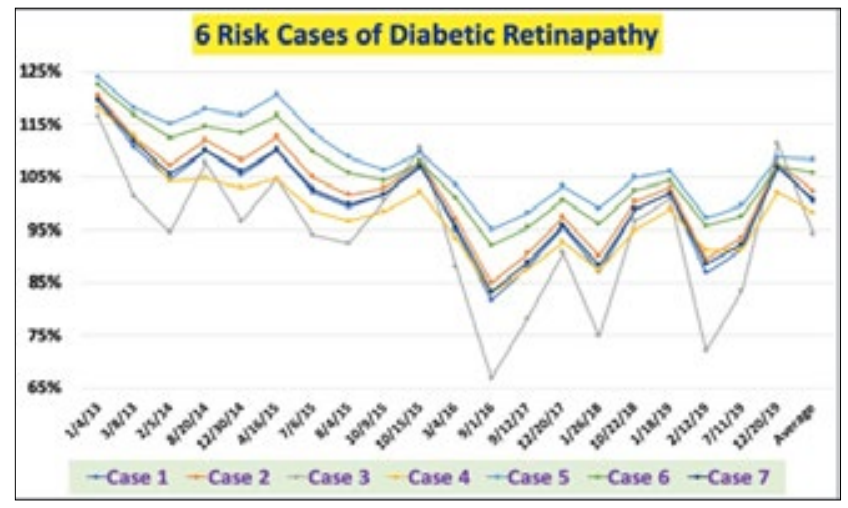

Figure 3: Time series Line Chart of diabetic retinopathy risk probability $\%$ of 7 cases

\begin{tabular}{|c|c|c|c|c|c|c|c|}
\hline Risk Case & Case 1 & Case 2 & Case 3 & Case 4 & Case 5 & Case 6 & Case 7 \\
\hline $1 / 4 / 13$ & $119 \%$ & $121 \%$ & $116 \%$ & $118 \%$ & $124 \%$ & $122 \%$ & $120 \%$ \\
\hline $3 / 8 / 13$ & $111 \%$ & $113 \%$ & $101 \%$ & $113 \%$ & $118 \%$ & $117 \%$ & $112 \%$ \\
\hline $2 / 5 / 14$ & $105 \%$ & $107 \%$ & $94 \%$ & $104 \%$ & $115 \%$ & $112 \%$ & $106 \%$ \\
\hline $8 / 20 / 14$ & $110 \%$ & $112 \%$ & $108 \%$ & $105 \%$ & $118 \%$ & $115 \%$ & $110 \%$ \\
\hline $12 / 30 / 14$ & $105 \%$ & $108 \%$ & $97 \%$ & $103 \%$ & $117 \%$ & $113 \%$ & $106 \%$ \\
\hline $4 / 16 / 15$ & $110 \%$ & $113 \%$ & $105 \%$ & $105 \%$ & $121 \%$ & $117 \%$ & $110 \%$ \\
\hline $7 / 6 / 15$ & $102 \%$ & $105 \%$ & $94 \%$ & $99 \%$ & $114 \%$ & $110 \%$ & $103 \%$ \\
\hline $8 / 4 / 15$ & $99 \%$ & $102 \%$ & $92 \%$ & $97 \%$ & $109 \%$ & $106 \%$ & $100 \%$ \\
\hline $10 / 9 / 15$ & $102 \%$ & $103 \%$ & $101 \%$ & $98 \%$ & $106 \%$ & $104 \%$ & $102 \%$ \\
\hline $10 / 15 / 15$ & $107 \%$ & $108 \%$ & $111 \%$ & $102 \%$ & $110 \%$ & $108 \%$ & $107 \%$ \\
\hline $3 / 4 / 16$ & $95 \%$ & $97 \%$ & $88 \%$ & $93 \%$ & $103 \%$ & $101 \%$ & $95 \%$ \\
\hline $9 / 1 / 16$ & $82 \%$ & $85 \%$ & $67 \%$ & $83 \%$ & $95 \%$ & $92 \%$ & $83 \%$ \\
\hline $9 / 12 / 17$ & $88 \%$ & $90 \%$ & $78 \%$ & $87 \%$ & $98 \%$ & $95 \%$ & $89 \%$ \\
\hline $12 / 20 / 17$ & $95 \%$ & $97 \%$ & $91 \%$ & $93 \%$ & $103 \%$ & $101 \%$ & $96 \%$ \\
\hline $1 / 26 / 18$ & $87 \%$ & $90 \%$ & $75 \%$ & $87 \%$ & $99 \%$ & $96 \%$ & $88 \%$ \\
\hline $10 / 22 / 18$ & $99 \%$ & $100 \%$ & $97 \%$ & $95 \%$ & $105 \%$ & $102 \%$ & $99 \%$ \\
\hline $1 / 18 / 19$ & $102 \%$ & $103 \%$ & $101 \%$ & $99 \%$ & $106 \%$ & $104 \%$ & $102 \%$ \\
\hline $2 / 12 / 19$ & $87 \%$ & $89 \%$ & $72 \%$ & $91 \%$ & $97 \%$ & $96 \%$ & $88 \%$ \\
\hline $7 / 11 / 19$ & $91 \%$ & $93 \%$ & $83 \%$ & $91 \%$ & $100 \%$ & $98 \%$ & $92 \%$ \\
\hline $12 / 20 / 19$ & $107 \%$ & $108 \%$ & $111 \%$ & $102 \%$ & $109 \%$ & $107 \%$ & $107 \%$ \\
\hline Average & $100 \%$ & $102 \%$ & $94 \%$ & $98 \%$ & $108 \%$ & $106 \%$ & $101 \%$ \\
\hline
\end{tabular}

Figure 4: Data Table of Diabetic Retinopathy risk probability $\%$ of 7 cases

\begin{tabular}{|c|c|c|c|c|}
\hline Risk Case & Risk $\%$ & Trig. & SBP & A1C \\
\hline Case 1 & $100 \%$ & $33 \%$ & $33 \%$ & $33 \%$ \\
\hline Case 2 & $102 \%$ & $30 \%$ & $30 \%$ & $40 \%$ \\
\hline Case 3 & $94 \%$ & $60 \%$ & $20 \%$ & $20 \%$ \\
\hline Case 4 & $98 \%$ & $20 \%$ & $60 \%$ & $20 \%$ \\
\hline Case 5 & $108 \%$ & $20 \%$ & $20 \%$ & $60 \%$ \\
\hline Case 6 & $106 \%$ & $20 \%$ & $30 \%$ & $50 \%$ \\
\hline Case 7 & $101 \%$ & $30 \%$ & $36 \%$ & $34 \%$ \\
\hline Case 7 & HR & 1.10 & 1.29 & 1.22 \\
\hline
\end{tabular}

Figure 5: Table of contribution weighting factors and risk probability $\%$ of 7 cases

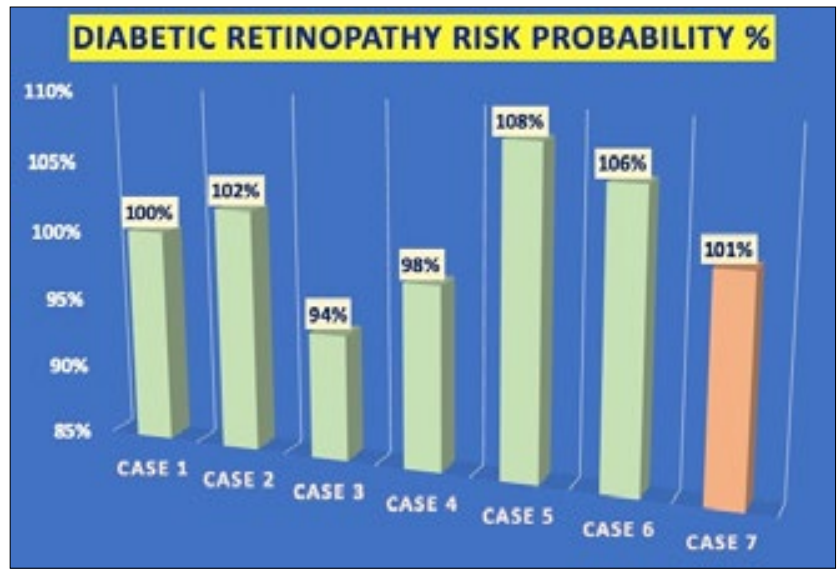

Figure 6: Bar Chart of diabetic retinopathy risk probability $\%$ of 7 cases 
Here is the list of these seven different weighting factors:

Case 1: Trig 33\%, SBP 33\%, A1C 33\% (even distribution at $33 \%$ each)

Case 2: Trig $30 \%$, SBP $30 \%$, A1C $40 \%$ (A1C is slightly heavier, $40 \%$ )

Case 3: Trig $60 \%$, SBP $20 \%$, A1C $20 \%$ (Trig is the heaviest, $60 \%)$

Case 4: Trig 20\%, SBP $60 \%$, A1C $20 \%$ (SBP is the heaviest, $60 \%)$

Case 5: Trig 20\%, SBP $20 \%$, A1C $60 \%$ (A1C is the heaviest, $60 \%)$

Case 6: Trig 20\%, SBP 30\%, A1C 50\% (A1C is heavier, 50\%)

Case 7: Trig 30\%, SBP 36\%, A1C 34\% (using Ref. 2's HR findings as a clue or source)

Figure 6 is a simplified Bar chart of risk probability $\%$ of these 7 -cases.

From Figures 3, 4, 5, 6 and 7, we can observe the following conclusive phenomena:

1. Upper bound: Both Case 5 (A1C 60\%) and Case 6 (A1C $50 \%$ ) have the highest risk probability \% (106\%-108\%).

2. Middle Ground: All of Case 7 (Ireland HR case), Case 1 (even distribution of 33\% each), and Case 2 (A1C 40\%) are within a range of moderate risk probability $\%(100-$ $102 \%)$.

3. Lower Bound: Both Case 3 (Trig 60\%) and Case 4 (SBP $60 \%$ ) have the lowest risk probability $\%(94-98 \%)$.

Based on the author's medical data and personal feelings of his diabetic retinopathy conditions at different progression stages, the upper bound curves, Case 5 and Case 6 , seem to be closely matched with the situation of his real conditions. On the opposite, the lower bound, Case 3 and Case 4, seem to be further apart from his real conditions.

Although lacking available medical test data, in general, he believes his overall diabetic retinopathy progression has slowed down for the past 7-years. His belief matched the trend of this set of curves.

Although both blood pressure and lipids share their responsibility of damaging the retina, based on this specific study, it seems that HbA1C plays a major role of his diabetic retinopathy conditions and developments.

In summary, he applied both linear regression analysis and Cox proportional-hazard model to calculate his "relative" risk probability $\%$ and his hazard ratio (HR) using 0.366 as its baseline. The comparison of these two approaches can be seen in Figure 7.

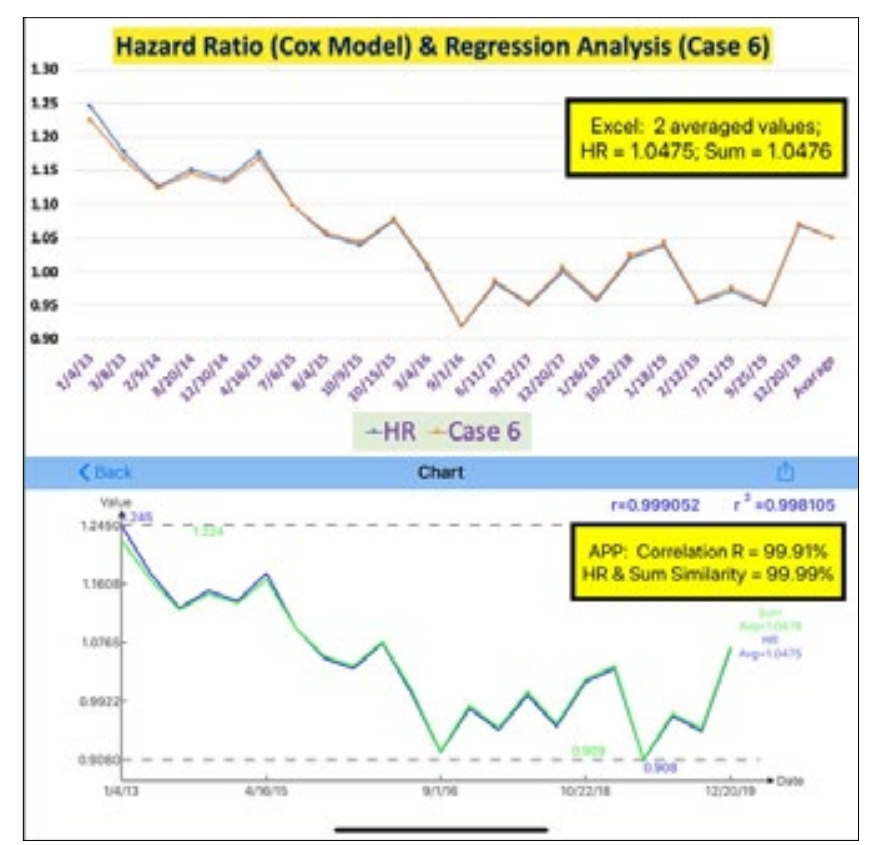

Figure 7: Comparison of Case 6 between weighted risk probability (Sum) \% and HR Model results

Although the absolute values are slightly different on certain test dates, these two curves have an extremely high correlation coefficient $(99.991 \%)$. These slight numerical differences are a result from HR Model utilizing exponential operation of the risk \% value. The most important thing is that they demonstrate the relative risk probability $\%$ and relative hazard ratio (HR) of an upper bound Case 6 with an extremely high correlation.

\section{Conclusion}

From the diabetic retinopathy sensitivity regression analysis results, it appears that diabetes, in particular $\mathrm{HbA1C}$, plays a more dominating role as the murderer, while both hypertension (SBP) and hyperlipidemia (Triglycerides) play supporting roles as the accomplices. It is no wonder that the medical community calls it diabetic retinopathy! Nevertheless, the combination of these three chronic diseases may definitely cause severe damage to the retina. Furthermore, as stated in Reference 1, diabetic retinopathy is not only a metabolic microvascular blood vessel issue, it is also a serious neuroscientific problem. The author strongly agrees with this viewpoint. In his recent research work of glucose, he has identified the amounts for both fasting and postprandial glucoses' production and timing are controlled by the brain and neuro-system. He has already proven previously that diabetes itself is also closely related to our brain and nervous system.

As pointed out in Reference 1, this particular study may shed some light on using an approach to strengthen metabolic conditions, combination of $\mathrm{HbA1C}$, SBP, and triglycerides in order to improve existing conditions of diabetic retinopathy in the patient. 
1. David A. Antonetti, Alistair J. Barber, Sarah K. Bronson1, Willard M. Freeman, Thomas W. Gardner, Leonard S. Jefferson, Mark Kester, Scot R. Kimball1, J. Kyle Krady, Kathryn F. LaNoue, Christopher C. Norbury, Patrick G. Quinn, Lakshman Sandirasegarane, Ian A. Simpson. (2006). Perspectives in Diabetes: Diabetic Retinopathy, Seeing Beyond Glucose-Induced Microvascular Disease. Diabetes. 55:2401-2411, 2006.doi.org/10.2337/db051635

2. J. J. Smith D. M. Wright P. Scanlon N. Lois. (2020). Risk factors associated with progression to referable retinopathy: a type 2 diabetes mellitus cohort study in the Republic of Ireland. Presented partially at the annual meeting of the Association for Research in Vision and Ophthalmology (ARVO), Vancouver, Canada, 28 April to 2 May 2019. doi.org/10.1111/dme. 14278

3. 3. Hsu, Gerald C. (2018). Using Math-Physical Medicine and Artificial Intelligence Technology to Manage Lifestyle and Control Metabolic Conditions of T2D. International Journal of Diabetes \& Its Complications, 2(3),1-7. Retrieved from http://cmepub.com/pdfs/ using-mathphysical-medicine-and-artificial-intelligencetechnology-to-manage-lifestyle-and-control-metabolicconditions-of-t2d-412.pdf

4. Hsu, Gerald C. (2018). A Clinic Case of Using MathPhysical Medicine to Study the Probability of Having a Heart Attack or Stroke Based on Combination of Metabolic Conditions, Lifestyle, and Metabolism Index. Journal of Clinical Review \& Case Reports, 3(5), 1-2. Retrieved from https:/www.opastonline.com/wp-content/ uploads/2018/07/a-clinic-case-of-using-math-physicalmedicine-to-study-the-probability-of-having-a-heartattack-or-stroke-based-on-combination-of-metabolicconditions-lifestyle-and-metabolism-index-jcrc-2018.pdf
Copyright: (2020 Gerald C. Hsu. This is an open-access article distributed under the terms of the Creative Commons Attribution License, which permits unrestricted use, distribution, and reproduction in any medium, provided the original author and source are credited. 